\title{
La dyslexie à l'âge adulte : la persistance des difficultés orthographiques
}

\author{
Audrey Mazur-Palandre ${ }^{1,2, \mathrm{i}}$ \\ ${ }^{1}$ Laboratoire ICAR (UMR5191, CNRS, Université Lyon 2 et ENS de Lyon), 15 parvis René \\ Descartes BP 7000, 69342 Lyon Cedex 07 \\ ${ }^{2}$ Laboratoire d'Excellence ASLAN, (ANR-10-LABX-0081, de l'Université de Lyon dans le \\ cadre du programme « Investissements d'Avenir » (ANR-11-IDEX-0007) de l'État Français \\ géré par l'Agence Nationale de la Recherche), 14 avenue Berthelot, 69363 Lyon Cedex 7
}

\begin{abstract}
Résumé. Dans cet article, les résultats préliminaires d'une étude plus large sur la production textuelle des étudiants dyslexiques sont présentés. Les productions écrites d'une vingtaine d'étudiants dyslexiques et contrôles sont analysées dans l'objectif de voir ce qu'il en est de la gestion de l'orthographe lexicale et grammaticale. En effet, lors d'analyses antérieures sur les mêmes populations, les étudiants dyslexiques se déclarent en difficultés lorsqu'il s'agit de rédiger un texte, et ce, que ce soit avec l'orthographe, la syntaxe ou l'organisation du texte lui-même. Un bilan orthophonique et neuropsychologique confirme que les étudiants dyslexiques ont encore des difficultés avec l'orthographe, la lecture et qu'ils présentent un fonctionnement attentionnel atypique. Pour cette présente étude, il a été demandé aux étudiants dyslexiques et contrôles de produire un texte narratif et un expositif, à l'écrit et à l'oral. Les versions écrites, dont il est question dans cet article, ont été collectées via le logiciel Eye and Pen $\odot$ puis transférées dans CLAN permettant le codage de toutes les erreurs d'orthographe. Les résultats révèlent que, si les étudiants dyslexiques et contrôles ont le même pattern d'erreurs, les dyslexiques font significativement plus d'erreurs que les contrôles. Une étude qualitative montre que certaines erreurs récurrentes des étudiants dyslexiques ne sont jamais relevées dans les textes des étudiants contrôles. Les étudiants dyslexiques ont encore de réelles difficultés avec l'orthographe.
\end{abstract}

\begin{abstract}
Dyslexia in adulthood: the persistence of orthographic difficulties. In this paper, the preliminary results of a larger study about the spoken and written production of dyslexic university students are presented. Written texts of dyslexic and control university students are analysed to see how they deal with lexical and grammatical spelling. In previous analyses with the same populations, dyslexic students report having still difficulties when they compose a written text, whether it be with spelling, syntax, or the organization of the text. A speech diagnostic and neuropsychological assessment confirm that dyslexic students still have difficulties with spelling, as with reading, and that they also have an atypical attentional functioning. For this present study, dyslexic students, as well as a group of paired control students, were asked to produce written and spoken narrative and expository texts. The written text, observed in this paper were collected using Eye and Pen (C) software
\end{abstract}


with graphics tablets and then transferred to CLAN software in which all errors were coded. Results reveal that, if dyslexic and control students have the same pattern of errors, dyslexics make significantly more errors than controls. A qualitative study shows that some recurring errors of dyslexic students are never found in the texts of the student controls. Dyslexic students still have real difficulties with spelling.

\section{Introduction}

Une bonne gestion de l'écrit est un atout fort dans notre société et apparait comme un gage de réussite, notamment scolaire. Toutefois, le chemin pour devenir un scripteur et un locuteur expert est bien long et l'individu dès son plus jeune âge doit apprendre à manipuler les contraintes et les exigences de l'oral et l'écrit, tout en faisant preuve de flexibilité et d'adaptation aux divers contextes de production. Si la production orale est acquise implicitement, sans enseignement, ce n'est pas le cas de l'écrit qui s'acquiert de manière explicite par le biais de l'instruction (Fayol, 1996) et qui est une tâche couteuse. Nous comprenons alors tout l'intérêt et l'importance des études se consacrant à la production, d'autant plus qu'il existe une réelle pression à gérer ces deux systèmes de manière performante. Cette pression, pouvant être génératrice d'angoisses (Fayol, 1996), est d'autant plus difficile à gérer lorsque les individus présentent des troubles spécifiques des apprentissages tels que la dyslexie, qui se définit par des difficultés à reconnaître rapidement les mots écrits et à les orthographier. Suite au Rapport Ringard (Ringard, 2000), signalant une prévalence importante de la dyslexie (6 - 8\%, Zorman, Lequette et Pouget, 2004), le Ministère de l'Éducation Nationale a lancé un Plan d'Action permettant de mieux dépister les élèves et de leur apporter des prises en charge adaptées. Néanmoins, la majorité des aides disparaissent dans l'enseignement supérieur, alors que de grandes difficultés persistent à l'âge adulte (Jacquier, Naudin, Roisin, Hoen et Meunier, 2009) et que les prises en charge, si elles réduisent les inconvénients, ne font pas entièrement disparaître les anomalies (Swanson et Hsieh, 2009). Les difficultés de décodage des mots et de récupération de leur orthographe en mémoire empêchent alors les dyslexiques de lire et écrire de manière fluide et de consacrer les ressources cognitives à comprendre et à élaborer des idées. Malgré les difficultés, les personnes dyslexiques arrivent à l'université et sont de plus en plus nombreuses. Ainsi, par exemple, à l'Université de Lyon, parmi les 969 étudiants en situation de handicap, $326(33,6 \%)$ présentent des troubles du langage ${ }^{\mathrm{ii}}$ : c'est dans ce contexte que des projets ${ }^{\mathrm{iii}}$, constitués d'équipes pluridisciplinaires, se consacrant à la dyslexie chez l'étudiant, ont été menés. Cette présente étude s'inscrit dans ces projets et se consacre à la production écrite d'étudiants dyslexiques et contrôles, et plus spécifiquement à la gestion du code orthographique : ce sont les premiers résultats, d'autres analyses, concernant les révisions, la cohérence et la cohésion étant en cours. C'est ainsi que les productions textuelles écrites d'une vingtaine d'étudiants dyslexiques et d'une vingtaine d'étudiants non dyslexiques appariés sont analysées, afin de voir ce qu'il en est de la gestion de l'orthographe lexicale et grammaticale chez ces individus présentant une dyslexie et scolarisés dans l'enseignement supérieur. Accéder aux études supérieures signifie-t-il que les étudiants en ont fini avec d'éventuelles difficultés ? Leur suivi orthophonique pendant l'enfance (primaire et collège) leur permet-il de contrer leurs troubles et de mettre en place des stratégies gommant totalement leurs difficultés?

Les enjeux sociétaux de la prise en charge des étudiants dyslexiques sont donc importants et doublés d'enjeux scientifiques car la connaissance des troubles persistants et les difficultés en résultant chez les jeunes adultes reste parcellaire. 


\section{Activité d'écriture et planification}

L'activité d'écriture est une des tâches cognitives les plus couteuses et complexes (Hayes, 1995, 1998 ; Kellogg, 1987, 1994 ; Olive et Piolat, 2005) impliquant, selon la version récente du modèle de Hayes sur la production textuelle à l'écrit, divers processus cognitifs tels que l'interprétation de texte (représentations internes des données linguistiques et graphiques, phénomènes de révisions), la réflexion, la résolution des problèmes (planification incluse), la prise de décision ou encore la production d'inférences (Hayes, 1998). Lors d'une production textuelle, le locuteur fait appel, entre autres, à des modèles mentaux structurés, à savoir « des représentations cognitives regroupant des informations et des connaissances relatives à une situation spécifique » (Hoc, 1992). Les individus doivent faire face à une situation paradoxale : le message doit être linéaire alors que le modèle mental est, par définition, multidimensionnel. Pour répondre à cette contrainte, les individus ont recours à trois stratégies de planification : la planification par connaissances rapportées, la planification par connaissances transformées (Bereiter et Scardamalia, 1987, 1998 ; Chanquoy et Alamargot, 2002) et la planification par connaissances révisées. La planification par connaissances rapportées correspond à un travail de récupération des contenus, des informations, pas à pas, dans la mémoire à long terme ; puis, ces derniers sont reformulés au fur et à mesure qu'ils sont récupérés « sans procéder à une réorganisation d'ensemble du contenu conceptuel ou de la forme linguistique du texte » (Chanquoy et Alamargot, 2002:7). Cette manière de procéder, constituer un texte pas à pas, permet aux enfants de produire un texte bien que la plupart des ressources cognitives sont consacrées à de multiples demandes de l'activité rédactionnelle telles que le travail grapho-moteur et la gestion du code orthographique. La planification par connaissances transformées appelle à un effort de mise en relation de deux espaces : celui des contenus et celui de la rhétorique (Bereiter et Scardamalia, 1987). Les connaissances récupérées en mémoire sont réorganisées en prenant en compte plus de paramètres linguistiques et pragmatiques. Selon les buts communicationnels, les locuteurs/scripteurs ajustent leur message (Chanquoy et Alamargot, 2002). La troisième stratégie, la planification par connaissances révisées (Kellogg, 2008), intègre la prise en considération des représentations mentales de l'interlocuteur.

Les jeunes enfants, contraints par les multiples demandes du processus rédactionnel « ne peuvent mettre en œuvre qu'une gestion pas à pas de leur production, en ne prenant en compte que certains paramètres rédactionnels [...] Le texte est donc élaboré sans réelle réorganisation des informations récupérées et de manière assez économique » (Chanquoy et Alamargot, 2002:7). En effet, jusqu'à environ 8-9 ans, les processus - de bas niveau chez l'adulte expert - de codage orthographique ou grapho-moteurs ne sont pas automatisés (Berninger et Swanson, 1994 ; Piolat, 2004). Pour devenir un scripteur-expert, l'une des premières étapes est donc la gestion du code orthographique et donc de la conversion phonème/graphème, qui reste ardue en français, langue relativement opaque dans laquelle les relations phonèmes/graphèmes sont inconsistantes (Fayol et Jaffré, 2005). Les ressources cognitives sont alors mobilisées pour gérer ces processus, et la planification se limite alors à l'enchaînement des propositions et celui des révisions à des corrections dites strictement locales (Berninger et Swanson, 1994 ; Piolat, 2004), ce qui affecte l'écrit des enfants (Fayol et Miret, 2005). Ceci se caractérise par la mobilisation de la planification par connaissances rapportées. La pratique et l'expérience permettent d'automatiser certains processus (comme la conversion phonème/graphème), exigeant moins de ressources cognitives, allouées alors à des processus de haut niveau (comme ceux des révisions). Que se passe-t-il pour des individus adultes dyslexiques ne pouvant automatiser totalement cette conversion?

Si les études sur le jeune adulte dyslexique restent peu nombreuses (Cavalli, 2016), quelques travaux peuvent être cités démontrant que les adultes dyslexiques ont encore de 
grandes difficultés dans le domaine de l'écrit (Giménez, Luque, Lopez-Zamora, FernandezNavas, 2015 ; Jacquier et al., 2009 ; Mazur-Palandre, Abadie et Bedoin, 2016). Les travaux de Gimenez et al. (2015), par exemple, révèlent que les étudiants dyslexiques se plaignent significativement plus que les autres étudiants de mélanger les lettres dans les mots, de confondre des mots en lecture et en écriture, d'avoir du mal à prendre des notes et d'être constamment obligés de vérifier l'orthographe lorsqu'ils écrivent tout en faisant quand même trop de fautes. Les conclusions d'une étude récente (Mazur-Palandre et al., 2016) vont dans ce sens en montrant que les plaintes des étudiants dyslexiques concernent la prise de notes, la gêne due au bruit en cours, la compréhension de consignes écrites lors des examens (même s'ils n'ont pas de déficit de compréhension), la production textuelle (de la gestion de l'orthographe à l'organisation du texte), et l'apprentissage de l'anglais. Les résultats des bilans cognitifs confirment la persistance de grandes difficultés chez les étudiants dyslexiques que ce soit en dictée de mots ou de textes. Néanmoins aucune de ses études ne propose de voir ce qu'il en est de la gestion du code orthographique en production textuelle.

\section{Méthodologie}

\subsection{Participants}

La collecte des données a été réalisée dans le cadre de projets sur la dyslexie dans l'enseignement supérieuri ${ }^{\mathrm{ii}}$. Un questionnaire en ligne sur les difficultés et besoins dans l'enseignement supérieur a été rempli par environ 1500 étudiants. Les réponses de 97 étudiants dyslexiques et 97 étudiants contrôles ont été sélectionnées pour réaliser des analyses, afin de mieux situer les difficultés et besoins des étudiants dyslexiques. Parmi ces étudiants, une trentaine d'étudiants de chaque groupe ont accepté de passer un bilan orthophonique et neuropsychologique, puis une tâche de production textuelle, dont il est question dans cet article.

Les données textuelles de 21 étudiants dyslexiques et 22 contrôles appariés en âge (Tableau 1), genre et niveau d'étude $(\mathrm{BAC}+2$ au $\mathrm{BAC}+5)$, ont été retenues pour les analyses (certaines ont dû être écartées pour diverses raisons techniques).

Tableau 1. Description des groupes d'individu.

\begin{tabular}{|l|c|c|}
\hline & Étudiants dyslexiques & Étudiants contrôles \\
\hline$\hat{\text { Âge }}$ & & \\
\hline Moyenne & 21,7 & 21,8 \\
\hline Intervalle & {$[18 ; 1-28 ; 5]$} & {$[18 ; 1-28 ; 9]$} \\
\hline Nombre total d'individus & $\mathbf{2 1}$ & $\mathbf{2 2}$ \\
\hline Nombre de filles & 9 & 10 \\
\hline Nombre de garçons & 12 & 12 \\
\hline
\end{tabular}

Les individus dyslexiques retenus, diagnostiqués durant l'enfance ${ }^{\mathrm{iv}}$, ont tous une dysorthographie associée et ont suivi au cours de leur enfance/adolescence des rééducations. Lors de la collecte des données, seuls deux étudiants dyslexiques parmi les 21 participants, ont dit être déclarés à la Mission Handicap de leur établissement et ainsi bénéficiés d'un tiers de temps durant les examens (aucun ne dispose donc d'outils numériques spécifiques ou ne suit un programme de remédiation au moment de la collecte). Les étudiants sont tous monolingues et francophones natifs. Ils ont tous signé un formulaire de consentement pour participer au bilan et à la tâche psycholinguistique. Les critères d'exclusion, vérifiés à l'anamnèse (lors du bilan), écartaient les individus présentant des déficits auditifs ou visuels, ou d'autres troubles. 


\subsection{Collecte des données}

\subsubsection{L'enquête}

Un premier objectif était de connaître les besoins et difficultés des étudiants dyslexiques de l'Université de Lyon. C'est dans cette mesure qu'une enquête sous forme de questionnaire en ligne a été élaborée ${ }^{v}$ et diffusée (mai 2014 à août 2014). Diverses questions ont été posées sur des thématiques allant de la vie étudiante aux apprentissages en passant par le diagnostic. Parmi les étudiants ayant participé, 97 étudiants dyslexiques et 97 étudiants contrôles appariés (genre, âge, niveau scolaire) ont été sélectionnés. Des tests statistiques ont été effectués sur chacune des questions afin de voir ce qui relève d'une difficulté commune à la majorité des étudiants et ce qui relève de difficultés plus spécifiques aux dyslexiques (pour une présentation des résultats , cf. Mazur-Palandre et al., 2016).

\subsubsection{Le bilan}

Plusieurs étudiants ayant répondu à l'enquête (dyslexiques et non dyslexiques) ont participé à un bilan orthophonique et neuropsychologique (pris en charge par une neuropsychologue ${ }^{\mathrm{vi}}$ et une psychologue ${ }^{\text {vii }}$ ) et qui débutait par une anamnèse par entretien individuel. Les suspicions de troubles neuro-développementaux (autre que la dyslexie-dysorthographie), neurologiques et psychiatriques, parfois associés à la dyslexie, ont été réfutées chez les étudiants retenus, qui ont également passé deux questionnaires : le DSM-V (American Psychiatric Association, 2013) et l'ASRS (Kessler, Adler, Ames, Demler, Faraone, Hiripi, Howes, Jin, Secnik, Spencer, Bedirhan Ustun, et Walters, 2005) (permettant d'écarter les cas de probable TDAH). Le traitement du langage écrit était évalué avec des épreuves de la batterie ECLA 16+ (Gola-Asmussen, Lequette, Pouget, Rouyer et Zorman, 2010) et du Vol du PC (Boutard, Claire et Gretchanovsky, 2004). Le décodage a ainsi été testé avec la lecture de mots isolés, la lecture à haute voix de textes avec et sans véritable signification (Le Vol du PC ; L'Alouette). L'orthographe était évaluée en dictée de mots isolés et de texte (ECLA 16+). La compréhension en lecture était mesurée à l'aide de subtests du Vol du PC (résumer le texte, questions ouvertes, etc). Enfin, les compétences métaphonologiques étaient évaluées avec la Suppression de Phonème Initial, les Contrepèteries et la Répétition de Pseudo-mots. Des tests neuropsychologiques issus de la TAP-M Zimmermann et Fimm, 2012) estimaient plusieurs aspects de l'attention et des fonctions exécutives : l'impulsivité (épreuve de Go/Nogo pour l'inhibition de réponses motrices automatisées), la distractibilité (inhiber des distracteurs visuels pour réaliser une tâche visuelle) et l'attention soutenue (se concentrer 15 minutes sur une tâche cognitivement coûteuse). Enfin, des compétences visuo-attentionnelles étaient évaluées avec le test du Report Global pour l'empan visuo-attentionnel (Bosse, Tainturier et Valdois, 2007 ; Bosse et Valdois, 2009 ; Valdois, Guinet, et Embs, 2014, pour EVADYS], le test SIGL pour les traitements global/local (Bedoin et Melina, 2014), un test de recherche visuelle (Barrage de $\mathrm{n}$, ECLA 16+) et des épreuves d'orientation visuelle et auditive (Visioner et Audioner, Bedoin, 2014). Deux épreuves des échelles de Wechsler évaluaient le raisonnement perceptif (Matrices), la mémoire à court terme et la mémoire de travail auditivo-verbales (Mémoire des chiffres) (pour une présentation des résultats , cf. Mazur-Palandre et al., 2016). 


\subsubsection{La passation psycholinguistique}

Durant la tâche psycholinguistique, il a été demandé aux étudiants de produire quatre textes à partir d'une vidéo muette sur la thématique du conflit ${ }^{\text {viii }}$, qui correspondent à quatre conditions expérimentales : narratif oral, narratif écrit, expositif oral et expositif écrit. La collecte s'est organisée en deux sessions séparées d'une semaine. Chaque participant a donc eu deux rendez-vous. Lors du premier (Session 1), la vidéo a été projetée aux individus qui ont continué en produisant deux textes séparés par une tâche de distraction. Lors du second rendez-vous (Session 2), les participants sont directement passés à la production de deux nouveaux textes, séparés par une tâche de distraction. Ils ont été répartis de manière équilibrée en deux ordres de passage (A ou B ; Tableau 2).

Tableau 2. Ordre de passage.

\begin{tabular}{llll}
\hline Ordre & Session 1 & Session 2 \\
\hline A & Visionnage & Oral - Questionnaire - Écrit & Oral - Questionnaire - Écrit \\
B & de la vidéo & Écrit - Questionnaire - Oral & Écrit - Questionnaire - Oral \\
\hline
\end{tabular}

L'ordre a été contrôlé car des études ont révélé son effet significatif pour certains critères d'analyse [entre autres, Jisa, 2004 ; Mazur-Palandre et Jisa, 2013). Le type de texte a également un important effet significatif pour de nombreux critères d'analyses. Par définition, le texte narratif et le texte expositif s'opposent par leurs structures, leurs contenus et leurs buts communicationnels (Mosenthal, 1985). Le type de texte narratif (personnel en monologue), qui se définit par une organisation temporelle et causale (Berman et Slobin, 1994 ; Mazur-Palandre et Jisa, 2006) ce qui facilite la mise en texte d'un événement (Fayol, 1997), sollicite un agencement de souvenirs personnels. Ceci se traduit alors par l'utilisation de formes spécifiques, de noms concrets, de clauses simples. Le texte expositif, quant à lui, est une production dans laquelle les locuteurs créent une structure thématique (Boscolo, 1990 ; Britton, 1994) et fait appel à des informations d'ordre générique [35]. Ce type de texte implique une pression informative et académique forte. Ceci se concrétise par l'utilisation de formes génériques, de noms abstraits, de structures syntaxiques complexes et de lexique de haut registre (Ravid, 2005).

\subsection{Transcription et codage des données}

\subsubsection{Transcription des données}

Les données orales ont été collectées par le biais d'enregistreur audio et de caméra vidéo. Elles ont ensuite été numérisées puis ont été transcrites sous le logiciel Transcriber, qui permet une reconnaissance des pauses silencieuses et donc un pré-découpage des données en silence versus bruit. Les transcriptions ont été réalisées en respectant les conventions CHILDES (Child Language Data Exchange System, http://childes.psy.cmu.edu/) afin de pouvoir, par la suite, les exporter, grâce à un filtre adapté $^{\text {ix }}$, dans le logiciel CLAN.

Les données écrites ont été collectées par le biais de tablettes graphiques via le logiciel Eye and Pen $($ ) (Chesnet et Alamargot, 2005). Eye and Pen $(\mathbb{C}$ a été conçu afin que les chercheurs en sciences cognitives et en linguistique puissent étudier les processus en temps réel lors de la production écrite. Ce logiciel peut enregistrer à la fois l'activité d'écriture et à l'activité oculaire. Pour notre étude, nous n'utilisons que la partie Pen du logiciel. Le texte est digitalisé par le biais de la tablette, puis affiché sur l'écran de l'ordinateur auquel la tablette est reliée. Ainsi, il est possible de reconstituer à l'écran et à volonté la trace laissée 
par le stylet. Les données écrites ont été transcrites puis pré-codées dans ce logiciel, comme les données orales, ont été exportées, grâce à un filtre adapté ${ }^{\mathrm{x}}$, dans le logiciel CLAN.

Le traitement sous CLAN. Une fois les données orales et écrites exportées dans le logiciel CLAN, ces deux types de production ont pu être codées et traitées de la même manière, selon les conventions CHILDES (Child Language Data Exchange System, http://childes.psy.cmu.edu/). Les productions ont été découpées en clauses et en unités terminales (UT, clause principale + clause(s) dépendante(s)) qui apparaissent comme de bons critères pour l'évaluation du développement syntaxique (Berman, 1998 ; Hunt, 1970 ; Ravid, 2005).

\subsubsection{Le codage des erreurs}

Marquillo (2003) propose une trame d'observation d'un texte distinguant quatre niveaux : le niveau pragmatique (adéquation de la production par rapport à la consigne, respects des modalités énonciatives, autonomie du texte, etc.); le niveau textuel et transphrastique (cohérence, progression de l'information, gestion de la ponctuation, etc.) ; le niveau de la phrase (ordre des mots, construction des verbes, syntaxe, etc.) ; et le niveau infraphrastique (correspondances phonie/graphie, etc). L'objectif à long terme est de pouvoir analyser tous ces niveaux dans les données afin de prendre en considération à la fois les dimensions textuelles et orthographiques de l'acte d'écriture.

Pour cet article, le focus est mis sur le niveau infraphrastique, et tout particulièrement sur les erreurs, qui peuvent être de type sémiographique ou phonographique selon le mécanisme dont elles relèvent. En effet, selon Jaffré (2000:1), on peut considérer que :

"l'écriture repose en fait sur un double principe. Le premier joue le rôle d'une mécanique de base qui assemble, selon des lois combinatoires, une série d'unités issues d'un inventaire restreint. Ces unités sont, dans tous les cas, déterminées par homologie avec des unités phonologiques (syllabes ou phonèmes) et relèvent par conséquent d'une phonographie. Toutefois, la raison d'être de l'écriture n'étant pas de noter des sons mais de représenter des signes, en l'occurrence des signes linguistiques, cette mécanique sert à la construction d'un autre principe de l'écriture, tout aussi essentiel que le premier, mais situé cette fois sur un plan qui englobe l'écriture d'unités lexicales, ou logographie, et l'écriture d'unités grammaticales, ou morphographie. C'est ce que nous appelons sémiographie.»

En nous inspirant de travaux antérieurs (Bétrix-Kohler, 1993 ; Catach, 1986; Catach, Gruaz, Duprez, 2008 ; Ducard, Honvault, Jaffré, 1995 ; De Weck et Fayol, 2009) parmi les erreurs sémiographiques, ont été distinguées : (1) les erreurs morphogrammiques lexicales, qui correspondent au non respect des morphogrammes lexicaux. Il s'agit des erreurs liées aux affixes mal orthographiés (singulièremant), aux lettres finales justifiables par dérivations (Parends) ou encore des erreurs relevant d'une non-reconnaissance des mots (enéfet); (2) les erreurs qui concernent les graphèmes non-fonctionnels, les doubles consonnes, ainsi que les lettres finales n'ayant pour explication que la diachronie (longtemp) ; (3) les erreurs de grammaire (morphogrammes grammaticaux), qui portent sur les marques de nombre (des guerre_), de genre (une personne caractériel_) et sur les terminaisons verbales (J'ait pu partir de chez moi); (4) les erreurs à dominante logogrammique, qui ont trait à la figure des mots permettant une distinction entre homophones ( $d \hat{u}$ et $d u / \grave{a}$ et $a /$ ses et ces) ; (5) les erreurs liées aux idéogrammes, qui portent donc sur les traits d'union, majuscules, les apostrophes ajoutées ou oubliées ; (6) les erreurs de segmentation (Par ce que).

Parmi les erreurs phonographiques, ont été distinguées: (1) les erreurs dites à dominante phonétique, contenant les oublis de graphèmes (mercedi), les confusions de graphèmes (correspandant) ainsi que les inversions (quarte au lieu de quatre) et adjonctions (quartre) ; (2) les erreurs dites à dominante phonogrammique, provenant d'une 
non connaissance du code écrit. Il y a d'une part, les erreurs altérant la valeur phonique du mot (saussise) et d'autre part les erreurs n'altérant pas la valeur phonique du mot (diffissile). Les erreurs d'accents ont été répertoriées dans cette catégorie.

\subsubsection{Méthode des juges}

Les données ont été transcrites, pré-codées et découpées en clauses par un transcripteur puis relues et corrigées par un second transcripteur. Un troisième transcripteur a vérifié le découpage des clauses et des UT. Les cas problématiques étaient abordés en réunion et une décision était prise par l'équipe.

Concernant le codage des données, un premier codeur a codé les données qui ont été ensuite recodées par un second codeur. Comme pour la transcription et le découpage en clauses et TU, les cas problématiques étaient abordés en réunion et une décision était prise par l'équipe.

Puis, afin d'établir une fiabilité concernant le codage des données, le travail des deux premiers codeurs a été mis en relation avec le codage d'un troisième codeur. Ce dernier a travaillé sur 14\% des données, à savoir douze textes: six textes expositifs (dont trois d'étudiants dyslexiques et trois d'étudiants non dyslexiques) et six textes narratifs (dont trois d'étudiants dyslexiques et trois d'étudiants non dyslexiques). Ces douze textes comportaient en tout 194 erreurs et les codeurs étaient en désaccord pour 8 erreurs. L'accord entre les codeurs étaient donc de $96 \%$.

\section{Hypothèses}

Notre hypothèse générale est que la gestion, à la fois de la structuration du texte et à la fois du système de conversion phonème/graphème, est cognitivement couteuse, et d'autant plus couteuse si les individus n'ont pas automatisé ou pas suffisamment le code orthographique du français. Ainsi, nous faisons les hypothèses suivantes :

- H1. Étant données les difficultés persistantes des étudiants dyslexiques, nous pensons que les étudiants dyslexiques font encore beaucoup d'erreurs en production de textes écrits, et ce même s'ils n'ont pas de contrainte de temps pour réaliser la tâche et que cette tâche est semi-guidée (la thématique est imposée mais les individus produisent librement). Ils en font, de plus, significativement plus que les étudiants contrôles, qui ont totalement automatisé le code orthographique.

- H2. Nous nous attendons à ce que les étudiants dyslexiques fassent plus d'erreurs dans les textes expositifs, qui impliquent une pression rédactionnelle forte, l'utilisation d'un lexique de registre élevé, que dans les textes narratifs.

- H3. Dans leur travaux, De Weck et Fayol (2009) montrent que les enfants, selon leur âge et s'ils sont dysorthographiques ou non, ont un pattern d'erreurs différent, nous supposons qu'il en est de même chez les étudiants de notre étude.

\section{Résultats}

Des analyses de variance (comparaison de moyennes, ANOVA) ont été effectuées avec : (a) deux facteurs interindividuels, le GROUPE (étudiants dyslexiques et contrôles) et l'ORDRE (oral/écrit et écrit/oral) ; et (b) un facteur intra-individuel, le TYPE DE TEXTE (expositif et narratif). Les interactions n'étant pas significatives ne sont pas présentées. 


\subsection{Nombre moyen d'erreurs par texte}

Un test ANOVA a été effectué sur le nombre moyen d'erreurs par texte, selon les facteurs GROUPE, TYPE DE TEXTE et ORDRE (Tableau 3).

Tableau 3. Résultats de ANOVA- nombre moyen d'erreurs par texte selon les facteurs GROUPE, TYPE DE TEXTE et ORDRE ${ }^{\mathrm{xi}}$

\begin{tabular}{|c|c|c|c|c|c|c|}
\hline Facteurs & $\mathrm{N}=$ & & Moyennes & Écart-type & ANOVA & \\
\hline \multirow[t]{2}{*}{ GROUPE } & \multirow[t]{2}{*}{43} & Dys. & 22,72 & 20,6 & \multirow{2}{*}{$F(1,39)=20,276, p=.001$} & \multirow[t]{2}{*}{$\mathbf{S}$} \\
\hline & & Contrôle & 4,65 & 3,75 & & \\
\hline \multirow[t]{2}{*}{ TYPE DE TEXTE } & \multirow[t]{2}{*}{43} & Expo. & 13,39 & 17 & \multirow[t]{2}{*}{$\mathrm{F}(1,39)=0,060, \mathrm{p}=.807$} & \multirow[t]{2}{*}{ NS } \\
\hline & & Narr. & 13,58 & 16,8 & & \\
\hline \multirow[t]{2}{*}{ ORDRE } & \multirow[t]{2}{*}{43} & $\mathrm{E} / \mathrm{O}$ & 16,5 & 17,25 & \multirow[t]{2}{*}{$\mathrm{F}(1,39)=1,735, \mathrm{p}=.196$} & \multirow[t]{2}{*}{ NS } \\
\hline & & $\mathrm{O} / \mathrm{E}$ & 10,87 & 16 & & \\
\hline
\end{tabular}

Les résultats montrent que le facteur GROUPE a un effet significatif sur la variable nombre moyen d'erreurs par texte : les étudiants dyslexiques font significativement plus d'erreurs (en moyenne 22,72 erreurs par texte) que les étudiants contrôles (en moyenne 4,65 erreurs par texte). Les facteurs TYPE DE TEXTE et ORDRE n'ont pas d'effet significatif.

\subsection{Types d'erreurs}

\subsubsection{Erreurs sémiographiques / erreurs phonographiques}

Les étudiants dyslexiques, comme les étudiants contrôles, font plus d'erreurs de type sémiographique que de type phonographique. Néanmoins, les nombres moyens d'erreurs sémiographiques, comme phonographiques, sont beaucoup plus importants dans les productions des étudiants dyslexiques que dans celles des étudiants contrôles :

- $\quad$ dans les textes expositifs, les étudiants dyslexiques font, par texte, en moyenne 18 erreurs sémiographiques (écart-type $=14,9$; contre 2,9 erreurs par texte pour les étudiants contrôles, écart-type $=2,5$ ) et seulement 5,3 erreurs phonographiques (écart-type $=6,5$; contre 1,1 erreurs pour les étudiants contrôles, écart-type $=1,5$ ) ; dans les textes narratifs, les étudiants dyslexiques font, part texte, en moyenne 17 erreurs sémiographiques (écart-type $=16,8$; contre 4,27 erreurs pour les étudiants contrôles, écart-type $=3,9$ ) et seulement 5,2 erreurs phonographiques (écart-type $=5,1 ;$ contre 1,1 erreurs pour les étudiants contrôles, écart-type $=0,9$ ).

\subsubsection{Les erreurs sémiographiques}

Un test ANOVA a été effectué sur le nombre moyen d'erreurs sémiographiques par texte, selon les facteurs GROUPE, TYPE DE TEXTE et ORDRE (Tableau 4).

Tableau 4. Résultats de ANOVA- nombre d'erreurs sémiographiques par texte selon les facteurs GROUPE, TYPE DE TEXTE et ORDRE

\begin{tabular}{|c|c|c|c|c|c|c|}
\hline Facteurs & $\mathrm{N}=$ & & Moyennes & Écart-type & ANOVA & \\
\hline \multirow[t]{2}{*}{ GROUPE } & \multirow[t]{2}{*}{43} & Dys. & 17,50 & 15,85 & \multirow{2}{*}{$F_{(1,39)}=20,395, p=.001$} & \multirow[t]{2}{*}{$\mathbf{S}$} \\
\hline & & Contrôle & 3,6 & 3,2 & & \\
\hline \multirow[t]{2}{*}{ TYPE DE TEXTE } & \multirow[t]{2}{*}{43} & Expo. & 10,3 & 13,1 & \multirow[t]{2}{*}{$\mathrm{F}_{(1,39)}=0,105, \mathrm{p}>.05$} & \multirow[t]{2}{*}{ NS } \\
\hline & & Narr. & 10,5 & 13,4 & & \\
\hline \multirow[t]{2}{*}{ ORDRE } & \multirow[t]{2}{*}{43} & $\mathrm{E} / \mathrm{O}$ & 12,8 & 14,25 & \multirow[t]{2}{*}{$\mathrm{F}_{(1,39)}=1,838, \mathrm{p}>.05$} & \multirow[t]{2}{*}{ NS } \\
\hline & & $\mathrm{O} / \mathrm{E}$ & 8,17 & 11,4 & & \\
\hline
\end{tabular}


Les résultats montrent que le facteur GROUPE a un effet significatif sur la variable nombre moyen d'erreurs par texte : les étudiants dyslexiques font significativement plus d'erreurs (en moyenne 17,5 erreurs sémiographiques par texte) que les étudiants contrôles (en moyenne 3,6 erreurs sémiographiques par texte). Les facteurs TYPE DE TEXTE et ORDRE n'ont pas d'effet significatif sur cette variable.

\subsubsection{Les erreurs phonographiques}

Un test ANOVA a été effectué sur le nombre moyen d'erreurs phonographiques par texte, selon les facteurs GROUPE, TYPE DE TEXTE et ORDRE (Tableau 5).

Tableau 5. Résultats de ANOVA- nombre d'erreurs phonographiques par texte selon les facteurs GROUPE, TYPE DE TEXTE et ORDRE

\begin{tabular}{|c|c|c|c|c|c|c|}
\hline Facteurs & $\mathrm{N}=$ & & Moyennes & Écart-type & \multicolumn{2}{|l|}{ ANOVA } \\
\hline \multirow[t]{2}{*}{ GROUPE } & \multirow[t]{2}{*}{43} & Dys. & 5,26 & 5,75 & \multirow[t]{2}{*}{$F_{(1,39)}=13,264, p=.001$} & \multirow[t]{2}{*}{$\mathbf{S}$} \\
\hline & & Contrôle & 1,11 & 1,2 & & \\
\hline \multirow[t]{2}{*}{ TYPE DE TEXTE } & \multirow[t]{2}{*}{43} & Expo. & 3,16 & 5,1 & \multirow[t]{2}{*}{$\mathrm{F}_{(1,39)}=0,001, \mathrm{p}=.982$} & \multirow[t]{2}{*}{ NS } \\
\hline & & Narr. & 3,11 & 4,1 & & \\
\hline \multirow[t]{2}{*}{ ORDRE } & \multirow[t]{2}{*}{43} & $\mathrm{E} / \mathrm{O}$ & 3,75 & 4,3 & \multirow[t]{2}{*}{$\mathrm{F}_{(1,39)}=0,961, \mathrm{p}=.337$} & \multirow[t]{2}{*}{ NS } \\
\hline & & $\mathrm{O} / \mathrm{E}$ & 2,6 & 4,7 & & \\
\hline
\end{tabular}

Les résultats montrent que le facteur GROUPE a un effet significatif sur la variable nombre moyen d'erreurs phonographiques par texte: les étudiants dyslexiques font significativement plus d'erreurs (en moyenne 5,26 erreurs phonographiques par texte) que les étudiants contrôles (en moyenne 1,1 erreurs phonographiques par texte). Les facteurs TYPE DE TEXTE et ORDRE n'ont pas d'effet significatif sur cette variable.

\subsection{Erreurs sémiographiques : quels types?}

Les erreurs sémiographiques peuvent être de plusieurs types. Ont été distinguées : les erreurs morphogrammiques lexicales, les erreurs concernant les graphèmes nonfonctionnels, les erreurs de grammaire ou concernant les morphogrammes grammaticaux, les erreurs à dominante logogrammique et les erreurs liées aux idéogrammes (cf. la section 4.3.2 pour une description des erreurs). Les erreurs les plus faites :

- par les étudiants dyslexiques sont (par ordre décroissant): des erreurs grammaticales $(11,21$ en moyenne par texte, $\sigma=10,75)$, des logogrammiques $(2,75$ en moyenne, $\sigma=2,4)$, celles concernant les graphèmes non fonctionnels ( 1,43 en moyenne, $\sigma=0,7)$, des idéogrammiques $(1,35$ en moyenne, $\sigma=2)$, des erreurs de segmentation $(0,45$ en moyenne, $\sigma=0,8)$, et des erreurs morphologiques lexicales $(0,29$ en moyenne, 0,6$)$.

- par les étudiants contrôles sont : des erreurs grammaticales $(2,1$ en moyenne par texte, $\sigma=2,3)$, des logogrammiques $(0,5$ en moyenne, $\sigma=0,9)$, celles concernant les graphèmes non fonctionnels $(0,5$ en moyenne, $\sigma=2,1)$, des idéogrammiques $(0,3$ en moyenne, $\sigma=0,4)$, des erreurs morphologiques lexicales $(0,09$ en moyenne, 0,3$)$ et des erreurs de segmentation $(0,065$ en moyenne, $\sigma=0,1)$.

Les étudiants, dyslexiques comme contrôles, font en majorité des erreurs grammaticales, le nombre moyen des autres types d'erreurs étant beaucoup plus réduit. Néanmoins, le profil des erreurs commises n'est pas le même entre les deux groupes d'individus.

Des tests ANOVA ont été effectués sur le nombre moyen d'erreurs sémiographiques selon leur type par texte, selon les facteurs GROUPE, TYPE DE TEXTE et ORDRE (Tableau 6). 
Tableau 6. Résultats des tests de comparaisons de moyennes ANOVA

\begin{tabular}{|c|c|c|c|}
\hline $\begin{array}{l}\text { Facteurs } \\
\text { Type d'erreurs }\end{array}$ & GROUPE & TYPE DE TEXTE & ORDRE \\
\hline Grammaticales & $F_{(1,39)}=18,839, p=.001$ & $F_{(1,39)}=0,286, p=.596$ & $\mathrm{~F}_{(1,39)}=1,282, \mathrm{p}=.264$ \\
\hline Graphèmes non fonctionnels & $F_{(1,39)}=5,005, p=.031$ & $F_{(1,39)}=0,810, p=.374$ & $F_{(1,39)}=0,218, p>.05$ \\
\hline Idéogrammiques & $F_{(1,39)}=8,234, p=.007$ & $F_{(1,39)}=2,431, p=.127$ & $\mathrm{~F}_{(1,39)}=3,111, \mathrm{p}=.086$ \\
\hline Logogrammiques & $F_{(1,39)}=22,420, p=.001$ & $\mathrm{~F}_{(1,39)}=3,110, \mathrm{p}=.089$ & $\mathrm{~F}_{(1,39)}=0,730, \mathrm{p}=.398$ \\
\hline Morphogrammiques lex. & $\mathrm{F}_{(1,39)}=2,336, \mathrm{p}=.134$ & $\mathrm{~F}_{(1,39)}=1,736, \mathrm{p}=.195$ & $F_{(1,39)}=1,636, p=.208$ \\
\hline Segmentation & $F_{(1,39)}=5,027, p=.031$ & $\mathrm{~F}_{(1,39)}=2,766, \mathrm{p}=.104$ & $F_{(1,39)}=5,273, p=.027$ \\
\hline
\end{tabular}

Nous pouvons voir que pour chaque type d'erreurs, le facteur GROUPE a un effet significatif. Les étudiants dyslexiques réalisent plus d'erreurs sémiographiques, quel que soit le type, sauf les erreurs morphogrammiques lexicales, pour lesquelles les étudiants dyslexiques et contrôles en font presque le même nombre moyen par texte. Notons que pour les erreurs de segmentation, le facteur ORDRE a un effet significatif : les étudiants font plus ce genre d'erreurs lorsqu'ils produisent dans l'ordre écrit/oral que dans l'ordre oral/écrit. Les étudiants dyslexiques font : 0,8 erreur par texte $(\sigma=1,5)$ dans l'ordre E/O ; et 0,09 erreur par texte $(\sigma=0,2)$ dans l'ordre $\mathrm{O} / \mathrm{E}$. Les étudiants contrôles font 0,1 erreur par texte $(\sigma=0,6)$ dans l'ordre $\mathrm{E} / \mathrm{O}$; et 0,04 erreur par texte $(\sigma=0,2)$ dans l'ordre $\mathrm{O} / \mathrm{E}$.

Les analyses révèlent une interaction entre les facteurs GROUPE et ORDRE : les étudiants dyslexiques seraient significativement plus sensibles à l'effet de l'ordre de production que les étudiants contrôles, pour lesquels la différence de moyenne n'est pas significative.

\subsection{Erreurs phonologiques : quels types?}

Les erreurs phonographiques peuvent être de plusieurs types : les erreurs dites à dominante phonétique ayant un impact sur la valeur phonique du mot et les erreurs à dominante phonogrammique qui peuvent avoir ou pas un impact sur la valeur phonique du mot (cf. la section 4.3.2 pour une description des erreurs). Les erreurs les plus faites :

- par les étudiants dyslexiques sont : les erreurs phonogrammiques (4,4 en moyenne par texte, $\sigma=5,05)$ puis les phonétiques $(0,88$ en moyenne, $\sigma=1,45)$;

- par les étudiants contrôles sont : les erreurs phonogrammiques $(0,95$ en moyenne par texte, $\sigma=1,1)$ puis les phonétiques $(0,15$ en moyenne, $\sigma=0,55)$.

Des tests ANOVA ont été effectués sur le nombre moyen d'erreurs phonographiques par texte selon leur type, selon les facteurs GROUPE, TYPE DE TEXTE et ORDRE (Tableau 7).

Tableau 7. Résultats des tests de comparaisons de moyennes ANOVA

\begin{tabular}{|l|c|c|c|}
\hline \multicolumn{1}{|c|}{ Facteurs } & GROUPE & TYPE DE TEXTE & ORDRE \\
Type d'erreurs & & & \\
\hline A dominante phonétique & $\mathbf{F}_{(\mathbf{1}, \mathbf{3 9})}=\mathbf{6 , 9 7 7}, \mathbf{p}=\mathbf{. 0 1 2}$ & $\mathrm{F}_{(1,39)}=0,268, \mathrm{p}=.607$ & $\mathrm{~F}_{(1,39)}=1,270, \mathrm{p}=.377$ \\
\hline À dominante phonogrammique & $\mathbf{F}_{(\mathbf{1 , 3 9 )}} \mathbf{= 1 2 , 6 3 , \mathbf { p } = \mathbf { . 0 0 1 }}$ & $\mathrm{F}_{(1,39)}=0,13, \mathrm{p}=.911$ & $\mathrm{~F}_{(1,39)}=0,833, \mathrm{p}=.367$ \\
\hline
\end{tabular}

Les analyses révèlent que le facteur GROUPE a un effet significatif sur la variable nombre moyen d'erreurs phonographiques à dominante phonogrammique: les étudiants dyslexiques font significativement plus ce genre d'erreurs $(4,4$ erreurs en moyenne par texte) que les étudiants contrôles $(0,95$ erreurs en moyenne par texte $)$ et sur le nombre d'erreurs phonographiques à dominante phonétique (0,88 erreurs en moyenne par texte) que les étudiants contrôles ( 0,15 erreurs en moyenne par texte).

Les erreurs de type phonographiques à dominante phonogrammique sont divisées en deux catégories : (a) celles altérant la valeur phonique du mot cible et (b) celles n'altérant pas la valeur phonique du mot cible. Des tests de comparaison de moyennes ont été effectués sur ces variables, selon les facteurs GROUPE, TYPE DE TEXTE et ORDRE (Tableau 8). 
Tableau 8. Résultats des tests de comparaisons de moyennes ANOVA

\begin{tabular}{|l|c|c|c|}
\hline \multicolumn{1}{|c|}{ Facteurs } & GROUPE & TYPE DE TEXTE & ORDRE \\
\hline Type d'erreurs & & & \\
\hline Altérant la valeur phonique & $\mathbf{F}_{(\mathbf{1 , 3 9 )}}=\mathbf{7 , 6 4 5}, \mathbf{p}=\mathbf{. 0 0 9}$ & $\mathrm{F}_{(1,39)}=0,940, \mathrm{p}=.338$ & $\mathrm{~F}_{(1,39)}=0,853, \mathrm{p}=.362$ \\
\hline N'altérant pas la valeur phonique & $\mathbf{F}_{(\mathbf{1 , 3 9 )}}=\mathbf{1 2 , 7 7 2} \mathbf{p}=\mathbf{. 0 0 1}$ & $\mathrm{F}_{(1,39)}=1,122, \mathrm{p}=.296$ & $\mathrm{~F}_{(1,39)}=0,426, \mathrm{p}=.518$ \\
\hline
\end{tabular}

Les analyses révèlent que le facteur GROUPE a un effet significatif sur les deux variables :

- les erreurs phonographiques à dominante phonogrammique altérant la valeur phonique du mot cible (dyslexiques : 2,15 en moyenne ; $\sigma=3,3$ / contre 0,43 en moyenne ; $\sigma=0,7$, pour les contrôles) ;

- les erreurs phonographiques à dominante phonogrammique n'altérant pas la valeur phonique du mot cible (dyslexiques : 2,3 en moyenne ; $\sigma=2,6 /$ contre 0,5 en moyenne; $\sigma=0,7$, pour les contrôles) .

En revanche, les facteurs TYPE DE TEXTE et ORDRE n'ont pas d'effet significatif.

\section{Discussion}

L'objectif de notre projet est de pouvoir analyser le texte dans sa globalité en comparant les productions des étudiants dyslexiques et contrôles. Nous présentons, dans cet article, les résultats de la première étape des analyses des données psycholinguistiques : les erreurs.

Les analyses révèlent que le type textuel n'a jamais d'effet significatif sur les variables observées. Nous attendions un effet du contexte (H2) : les textes expositifs impliquant, entre autres, une contrainte normative et une pression textuelle plus importante que le texte narratif (Mazur-Palandre, 2015 ; Ravid, 2005) ou encore une structure moins clairement identifiée et connue que le texte narratif (Boscolo, 1990 ; Britton, 1994 ; Katzenberger, 2004), auraient pu contenir plus d'erreurs que les textes narratifs mais ce n'est pas le cas. Les étudiants dyslexiques, comme les contrôles, font autant d'erreurs dans les textes expositifs que dans les textes narratifs.

Concernant l'ordre de production (oral / écrit ou écrit / oral), les analyses révèlent que ce facteur a un effet significatif sur une seule variable: le nombre d'erreurs de segmentation (du type par ce que) : les étudiants dyslexiques (cf. l'interaction, section 5.4) font plus d'erreurs de segmentation (7) lorsqu'ils produisent d'abord à l'écrit et ensuite à l'oral. Ce résultat pourrait indiquer que le fait de passer d'abord à l'oral aiderait les étudiants dyslexiques à ensuite segmenter de manière correcte à l'écrit.

Le facteur groupe a un effet significatif pour bon nombre de variables. Les étudiants dyslexiques font significativement beaucoup plus d'erreurs que les étudiants contrôles (ce qui confirme notre hypothèse $\mathrm{H} 1$ ) et ceci est vrai pour les deux types d'erreurs : les erreurs sémiographiques (1) $)^{\mathrm{xii}}$ et les phonographiques (2).

\section{(1) Dans cette partit, de la vidéo toute les personnes qui triches sont associé a la couleur rouge (vetements, trousse).(34,TE)}

Dans cet exemple, l'étudiant dyslexique réalise plusieurs erreurs sémiographiques : une concernant un graphème non fonctionnel (partit), une logogrammique (a/à) et trois de grammaire en lien avec les marques de pluriel (toute, triches, associé).

\section{(2) Les non-dit, les sous-entendu négatifs peuvent blaiser l'autre. (02,TE)}

Dans cet exemple, l'étudiant dyslexique fait une erreur phonographique à dominante phonogrammique altérant la valeur phonique du mot (blaiser).

Quand nous regardons plus précisément les erreurs sémiographiques, les analyses révèlent que les étudiants dyslexiques font significativement plus d'erreurs morphogrammiques grammaticales (3), d'erreurs concernant les graphèmes non fonctionnels (4), d'erreurs 
idéogrammiques (5), logogrammiques (6) et de segmentation (7) que les étudiants contrôles.

(3) Et nous avions fait le choix après plusieur constation avec le professeur de travailler sur les moteur à deux temps (les moteurs de mobilette). Si_c'est moteur pouvait consomer moins de carburant avec une autre énergie, celui de l' éthanol. $(64, T N)$

L'étudiant dyslexique réalise plusieurs erreurs sémiographiques morphogrammiques grammaticales: des oublis d'accord en nombre dans les syntagmes nominaux et les syntagmes verbaux et une erreur de genre lors du remplacement du mot «énergie » par le pronom « celui ».

(4) Ils vont être réglés, du moins dans la plupart des cas grâce à un échange entre les béligérants. $(15, T E)$

L'étudiant dyslexique omet un «1» dans le mot «belligérants ».

(5) Lorsque du a coup je vois le garçons saisir la fille avec violence. je n'est pas pu rester spectateur de cette scène. $(76, T N)$

L'étudiant dyslexique ne met pas de majuscule au pronom «je », en début de phrase.

(6) À partir de ce moment la , quand une personne ne va pas dans notre intérêt (personne groupe évenement) un conflit apparait entre les personnes. $(05, T E)$

L'étudiant dyslexique ne met pas d'accent à « là », ce qui laisse à penser à une confusion entre le déterminant «la » et l'adverbe « là ».

(7) Biensur de doit m' occuper de tout le materielle. $(64, T N)$

L'étudiant ne segmente pas le mot «bien sûr».

En revanche, la différence de moyennes concernant les erreurs morphogrammiques lexicales entre les étudiants dyslexiques et les étudiants contrôles n'est pas significative.

Concernant les erreurs phonographiques, les analyses révèlent que les étudiants dyslexiques font significativement plus d'erreurs à dominante phonogrammique que les étudiants contrôles et ce que ce soit pour les erreurs altérant (8) ou pas (9) la valeur phonique du mot cible.

(8) La resolution de ses problèmes aura alors un processus different. $(15, T E)$

(9) Nous faisons notre maximum avec Denis pour favoriser la rencontre entre Amandine et Yohann, qui eux ne soupsonne rien. $(01, T E)$

Une analyse qualitative des erreurs phonographiques à dominante phonogrammique altérant la valeur phonique du mot cible, révèle que quand les étudiants contrôles font ce type d'erreurs, ce ne sont que des erreurs relevant de l'accentuation. Pour les étudiants dyslexiques, quand ils font ce type d'erreurs, nous retrouvons certes des erreurs liées aux accents mais également des erreurs traduisant encore des limites avec le code orthographique (9). Nombreux des étudiants dyslexiques ont une utilisation déviante des accents : ils peuvent les utiliser de manière très sporadique, et ce même si l'absence d'accents a un impact sur la valeur phonique du mot, ou se tromper d'accent.

De plus, les analyses révèlent que les étudiants dyslexiques font significativement plus d'erreurs phonographiques phonétiques (10) que les étudiants contrôles. Deux seulement sont relevées dans les productions des étudiants contrôles : «sourcilière » écrit « sourcillière » et « interaction » écrit « interation ». 
(10) Les situations de conflits peuvent être associé a des violance physique : bagare dans le couloir cette situation va engengrer différente réaction : igorance passage sans la remarquer, interomption par un adulte. $(34, T E)$

Ces analyses ont permis également de dresser le profil des erreurs des étudiants dyslexiques et contrôles, qui sont similaires (ce qui ne va pas dans le sens de l'hypothèse H3). Les étudiants dyslexiques tout comme les contrôles font, par ordre décroissant : des erreurs grammaticales, phonogrammiques, logogrammiques, des erreurs concernant les graphèmes non fonctionnels, des idéogrammiques, des phonétiques, des erreurs de segmentation et des erreurs morphologiques lexicales (pour ces deux derniers types d'erreurs, l'ordre s'inverse pour les étudiants contrôles). Les erreurs les plus fréquentes, pour les deux groupes, sont les erreurs grammaticales, phonogrammiques et logogrammiques. Les autres types d'erreurs sont plus marginaux, même si les différences de moyennes entre les deux groupes sont significatives (excepté pour les morphogrammiques lexicales), et donc à ne pas ignorer. Certaines erreurs sont donc beaucoup plus faites que d'autres, et ce même si les étudiants ne sont pas soumis à une contrainte de temps et même s'ils choisissent eux-mêmes leurs mots. Dans une étude sur des jeunes enfants, De Weck et Fayol (2009) ont montré que même s'il y avait une suprématie des erreurs concernant les morphogrammes grammaticaux puis des erreurs phonogrammiques, il pouvait être établi des profils d'erreurs différents selon l'âge des enfants et selon s'ils étaient dysorthographiques. Nos travaux confirment, en partie, ces conclusions : chez les étudiants, nous trouvons également une suprématie des erreurs concernant les morphogrammes grammaticaux et des erreurs phonogrammiques, même si les proportions chez les étudiants contrôles est dérisoires. Les analyses ne révèlent, en revanche, pas de profils distincts entre les deux groupes d'étudiants.

Malgré un profil similaire, une étude qualitative des erreurs grammaticales révèle que les étudiants dyslexiques font des erreurs que ne font jamais les étudiants contrôles. Prenons, par exemple, les erreurs concernant l'accord en nombre du verbe, certains étudiants dyslexiques peuvent faire des erreurs de type : utiliser la forme plurielle pour le singulier ("cette discordance ne peuvent être toléré») ou encore utiliser le pluriel du syntagme nominal pour une forme verbale en mettant « $\mathrm{s}$ » au lieu de «ent» («les personnes proviennes »), erreurs qui ne sont jamais relevées dans les textes des étudiants contrôles. Les étudiants contrôles vont, dans une moindre mesure, faire également quelques erreurs concernant l'accord en nombre du verbe, mais il s'agit seulement d'erreurs d'accord avec le participe passé, et principalement l'accord de l'objet direct avec le verbe avoir quand il est placé avant. Un second exemple est le pluriel des syntagmes nominaux : les étudiants dyslexiques peuvent oublier de mettre la marque du pluriel «s $»$ («ces conflit»), ce qui est arrivé seulement deux fois dans toutes les productions des étudiants contrôles. De plus, les étudiants dyslexiques peuvent faire l'erreur de marquer le pluriel des mots en « $\mathrm{au} »$ et «eau» avec un « $\mathrm{s} »$ au lieu d'un « $\mathrm{x} »$ ( «des pinceaus»), ce qui n'a pas été relevé dans les productions des étudiants contrôles. Un troisième exemple est celui des erreurs liées à la morphologie verbale : les étudiants dyslexiques peuvent faire des erreurs surprenantes, que nous n'attendons plus en études supérieures et que nous n'avons d'ailleurs pas retrouvées dans les textes des étudiants contrôles : j'apprend, tu comprend, je vu, j'expliqua, j'ai préférez, nous somme, il vas, j'était, elle racontais, nous seront, etc. Une caractéristique des étudiants dyslexiques est l'inconsistance -- un même individu dyslexique peut marquer correctement certains pluriels puis se tromper pour d'autres (10) -et, à la fois, la récurrence, en faisant ces mêmes types d'erreurs très fréquemment. Mentionnons que, si des difficultés persistent, certains étudiants dyslexiques s'en sortent mieux que d'autres, néanmoins leurs erreurs restent des erreurs atypiques, jamais réalisées par les contrôles. Des analyses complémentaires prenant en compte le degré de transparence des mots choisis et orthographiés par les étudiants dyslexiques et contrôles sont envisagées via l'utilisation de MANULEX (Lété, Sprenger-Charolles et Colé, 2004 ; 
Ortéga et Lété, 2010) : est-ce que les étudiants dyslexiques ont tendance à choisir des mots avec une orthographe à fort degré de transparence ? Est-ce que leur insécurité orthographique est liée au degré de transparence des mots?

Les étudiants dyslexiques de notre étude ont, en général, encore des difficultés en orthographe, ce qui va dans le sens des résultats des bilans orthophoniques et neuropsychologiques de cette même population (Abadie et Bedoin, 2016 ; Mazur-Palandre et al., 2016). Par exemple, en dictée de texte, les étudiants dyslexiques font plus d'erreurs que les étudiants contrôles, que ce soit pour les erreurs d'usages ou les erreurs d'accords. Si les analyses révélaient que les performances en lecture suggéraient un important déficit de l'application des règles grapho-phonologiques, les analyses du bilan sur les erreurs en dictée de texte et de mots isolés montrent que les règles phono-graphémiques sont un peu plus stables : les étudiants dyslexiques produisent des orthographes acceptables pour les pseudo-mots, et le fait de mobiliser les règles les plus courantes leur donne de bonnes chances d'orthographier les mots réguliers correctement mais orthographier les mots irréguliers reste particulièrement problématique (Abadie et Bedoin, 2016 ; Mazur-Palandre et al., 2016). Les règles associant phonèmes et graphèmes ne sont alors pas pertinentes et ils ont apparemment un déficit persistant d'accès au lexique orthographique. Les résultats en lecture de ces mêmes travaux suggéraient l'existence d'un certain lexique orthographique chez les étudiants dyslexiques de cette étude, et c'est pourquoi le déficit en production orthographique des mots irréguliers peut sans doute s'expliquer surtout par des difficultés à accéder rapidement et précisément à ce lexique, en l'absence de toute information orthographique, et donc en dictée, en prise de notes ou encore en production textuelle, comme c'est le cas dans ce présent article. Ceci pourrait expliquer le fait que les étudiants dyslexiques restent en difficulté, et ce, beaucoup plus que les contrôles, pour gérer les homophones (ce qui se traduit par de nombreuses erreurs logogrammiques) : un lexique orthographique instable et déficitaire ne fait, sans doute, qu'empirer le traitement de mots ayant une même prononciation mais un sens autre et une orthographe proche mais différente.

Il est admis que les jeunes enfants, n'ayant pas automatisé le codage orthographique et ainsi contraints à de multiples demandes liées au processus rédactionnel, ne peuvent constituer une production textuelle écrite, seulement de manière très basique, appelant une planification par connaissances rapportées (Bereiter et Scardamalia, 1987, 1998, 2014 ; Berninger et Swanson, 1994 ; Chanquoy et Alamargot, 2002 ; Piolat, 2004). Pour devenir un scripteur expert, il semble qu'une des premières étapes serait alors d'automatiser ce codage orthographique, afin de pouvoir allouer les ressources cognitives à des processus de plus haut niveau tels que la gestion de la macrostructure ou des révisons (planification par connaissances transformées et révisées). Les étudiants dyslexiques, s'ils ne peuvent totalement l'automatiser, sont capables néanmoins de produire des textes écrits. Des analyses en cours sur la syntaxe révèlent, par exemple, que les étudiants dyslexiques ont le même répertoire syntaxique que les étudiants contrôles. Néanmoins, les résultats préliminaires montrent qu'ils semblent «se perdre» dans certaines structures contenant différents niveaux syntaxiques (accumulation de subordonnées). Des futures analyses syntaxiques et macro-syntaxiques viendront rapidement complétées ces analyses sur le code orthographique, afin de pouvoir voir le réel impact de la non-automatisation totale du code orthographique, sur les processus rédactionnels dits de haut niveau, chez l'étudiants dyslexiques. Des analyses sont actuellement en cours sur les phénomènes de révisions. Morken et Helland (2013) concluent de leurs analyses que les jeunes enfants dyslexiques, lors d'une tâche de dictée de phrases, semblent réviser leur écrit autant que les enfants typiques ; la différence entre les enfants dyslexiques et typiques résiderait en une moins bonne qualité de la révision. Ainsi, qu'en est-il des étudiants dyslexiques ? Ont-ils le même fonctionnement de révision que les étudiants contrôles ? Des réponses à ces questions seront rapidement apportées. De plus, nous pouvons nous poser la question de l'impact des 
erreurs sur la production textuelle en général : est-ce que le fait de ne pas avoir automatisé totalement le code orthographique a un effet négatif sur la cohérence et la cohésion du texte? Analyser les textes des étudiants dans leur globalité (orthographe, syntaxe, cohérence, etc) permettra alors d'avoir une vision globale de la gestion textuelle des étudiants dyslexiques et de voir les processus rédactionnels qu'ils arrivent à gérer, malgré une non-automatisation totale du code orthographique, et ceux dont la gestion pose encore problème.

Nous tenons à remercier : Raphaëlle Abadie grâce à qui la collecte des données s'est parfaitement déroulée, la passation et l'analyse des bilans et pour son expertise en tant que neuropsychologue; Nathalie Bedoin pour son expertise en psychologie cognitive (notamment concernant la dyslexie); Florence Chenu pour, entre autres, son aide technique (création de filtres pour l'export dans CLAN, gestion du logiciel Eye and Pen()) ; Louis Maritaud pour son minutieux codage des données psycholinguistique ; Céline Faure (ISH) pour ses conseils statistiques ; Harriet Jisa (DDL UMR 5596) pour ses conseils avertis et ses relectures ; les étudiants volontaires ; le LabEx ASLAN (ANR-10LABX-0081) de l'Université de Lyon dans le cadre du programme "Investissements d'Avenir » (ANR-11-IDEX-0007) de l'État Français géré par l'Agence Nationale de la Recherche (ANR), le CNRS, le laboratoire ICAR (UMR 5191) et l'ENS de Lyon pour leur soutien financier.

\section{Références}

Abadie, R. et Bedoin, N. (2016). Les étudiants dyslexiques à l'université. Quels déficits cognitifs et langagiers? Neurologies, 192, 298-303.

Bedoin, N. (2014) Dyslexie chez l'enfant et déficits d'attention spatiale. Conférence invitée aux Entretiens d'Orthophonie 2014, Paris.

Bedoin, N. et Médina, F. (2014). Logiciel Sélection et Inhibition des traitements Global et Local, www.gnosia.fr.

Bereiter, C. et Scardamalia, M. (1987). The psychology of written composition. Hillsdale: Lawrence Erlbaum.

Bereiter, C. et Scardamalia, M. (1998). L'expertise en lecture-rédaction. In Piolat, A., Pélissier, A. (Eds.), La rédaction de textes, Approche cognitive, 13-50. Lausanne: Delachaux et niestlé.

Bereiter, C. et Scardamalia, M. (2014). Knowledge building and knowledge creation: One concept, two hills to climb. In S. C. Tan, H. J. So, J. Yeo (Eds.) Knowledge creation in education, 35-52. Singapore: Springer.

Berman, R. (1998). Typological perspectives on connectivity, In Penner, Z., Dittmar, N. (Eds.), Issues in the theory of language acquisition, 203-224. Bern: Peter Lang.

Berman, R. et Slobin, D. (1994). Relating events in narrative: A crosslinguistics developmental study. Hillsdall, NJ: Lawrence Erlbaum Associates.

Berninger, V. et Swanson, H. (1994). Modifying Hayes and Flower's model of skilled writing to explain beginning and developing writing. In Carlson, J., Buttterfly, E. (Eds.), Advances in Cognition and Educational Practice, Children's Writing: Toward a Process Theory of the Development of Skilled Writing, Vol. 2, 57-8. Greenwich: J.A.I. Press.

Bétrix-Kohler, D. (1993). Du bon usage de l'erreur dans une didactique de l'orthographe. In: L. Allal, D. Bain, P. Perrenoud (Eds.), Évaluation formative et didactique du français, 161-170. Neuchâtel: Delachaux et Niestlé.

Boscolo, P. (1990). The construction of expository text. First Language, 10, 217-230.

Britton, B. (1994). Understanding expository text: Building mental structures to induce insights. In Gernsbacher, M. (Eds.), Handbook of Psycholinguistics, 641-674. San Diego, California: Academic Press.

Bosse, M. L., Tainturier, M. J. et Valdois, S. (2007). Developmental dyslexia: The visual attention span deficit hypothesis. Cognition, 104(2), 198-230.

Bosse, M. L. et Valdois, S. (2009). Influence of the visual attention span on child reading performance: A cross-sectional study. Journal of Research in Reading, 32, 230-253.

Boutard, C., Claire, I., et Gretchanovsky. L. (2004) Le vol du PC : évaluation fonctionnelle de la lecture chez les sujets de 11 à 18 ans. Isbergues : Ortho Editions.

Catach, N. (1986). L'orthographe française. Traité théorique et pratique. Paris: Nathan. 
Catach, N. Gruaz, C. et Duprez, D. (2008). (3 ${ }^{\text {ème }}$ éd.). L'orthographe française. Traité théorique et pratique avec des travaux d'application et leurs corrigés. Paris: Armand.

Cavalli, E. (2016). La lecture de l'adulte dyslexique universitaire. Thèse de doctorat, Université AixMarseille.

Chanquoy, L. et Alamargot, D. (2002). Mise en place et développement des traitements rédactionnels : le rôle de la mémoire de travail. Le Langage et L'homme, 38, 171-190.

Chesnet, D. et Alamargot, D. (2005). Analyses en temps réel des activités oculaires et graphomotrices du scripteur: intérêt du dispositif 'Eye and Pen'. L'Année Psychologique, 105, 477-520.

De Weck, G. et Fayol, M. (2009). L'orthographe en production de textes chez les enfants avec et sans dysorthographie. Langage et pratiques, 43, 46-58.

Ducard, D., Honvault, R. et Jaffré, J.-P. (1995). L'orthographe en trois dimensions. Paris, Nathan.

Fayol. M. (1996). La production du langage écrit. In Jacques David et al. (Eds), L'apprentissage de l'écriture de l'école au collège, 7-36. Presses Universitaires de France " éducation et formation / L'éducateur $\gg$.

Fayol, M. (1997). Des idées au texte : psychologie cognitive de la production verbale orale et écrite. Paris : PUF.

Fayol, M. et Jaffré, J.-P. (2016). L'orthographe : des systèmes aux usages. Pratiques, Linguistiques, littérature, didactiques, en ligne, 169-170, mis en ligne le 30 juin 2016.

Fayol, M. et Miret, A. (2005). Écrire, orthographier et rédiger des textes, Psychologie Française, 50, 391-402.

Giménez, A., Luque, J. L., Lopez-Zamora, M. et Fernandez-Navas, M. (2015). A self-report of reading disabilities for adults: ATLAS. Anales de Psicologia, 31(1), 109-119.

Gola-Asmussen, C., Lequette, C., Pouget, G., Rouyer, C. et Zorman, M. (2010). Outil d'évaluation de compétences de lecture chez l'adulte de plus de 16 ans. Université de Provence Aix-Marseille 1 et Cognisciences LES Université Pierre Mendès.

Hayes, J. (1995). Un nouveau modèle du processus d'écriture, In Boyer, J.Y., Dionne, J.P. (Eds.), La production de textes : vers un modèle d'enseignement de l'écriture, 49-72. Montréal: Logiques.

Hayes, J. (1998). Un nouveau cadre pour intégrer cognition et affect dans la rédaction, In Piolat, A., Pélissier, A. (Eds.), la rédaction de textes, 51-101. Lausanne: Delachaux et Niestlé.

Hoc, J.M. (1992). Psychologie cognitive de la planification. Grenoble: Presse Universitaire de Grenoble Jacquier, C., Naudin, O., Roisin, A., Hoen, M., Meunier, F. (2009). Évaluation psychosociale de l'impact de la dyslexie à l'âge adulte. Colloque Neurosciences, Éducation et Francophonie (NEF), 24-26 mars, Lyon, France.

Hunt, K. (1970). Syntactic Maturity in School children and Adults, Monographs of the Society for Research in Child Development, 35, iii-67.

Jaffré, J.-P. (2000). Écritures et sémiographie, Linx, 43, 15-28.

Jisa, H. (2004). Growing into academic French. In Berman, R. (Eds.), Later Language Development: Typological and Psycholinguistic Perspectives, (TiLAR), 135-161. Amsterdam : John Benjamins.

Jisa, H. et Mazur, A. (2006). L'expression de la causalité : une étude développementale. Paper presented at the Journée d'étude : Des savoirs savants aux savoirs enseignés, 33-60. Université Paris X, Nanterre.

Katzenberger, I. (2004). The development of clause packaging in spoken and written texts, Journal of Pragmatics, 36, 1921-1948.

Kellogg, R. (1987) Effects of topic knowledge on the allocation of processing time and cognitive effort to writing processes, Memory and Cognition, 15, 256-266.

Kellogg, R. (1994). The psychology of writing. New York: Oxford University Press.

Kellogg, R. (2008). Training writing skills: A cognitive developmental perspective, Journal of, 1, 126.

Kessler, R. C., Adler, L., Ames, M., Demler, O., Faraone, S., Hiripi, E., Howes, M.J., Jin, R., Secnik, K., Spencer, T., Bedirhan Ustun, T. et Walters, E. E. (2005). The World Health Organization adult ADHD self-report scale: A short screening scale for use in the general population. Psychological Medicine, 2, 245-256.

Lété, B., Sprenger-Charolles, L., et Colé, P. (2004). Manulex: A grade-level lexical database from French elementary-school readers. Behavior Research Methods, Instruments, \& Computers, 36, 156-166.

Marquillo, M. (2003). L'interprétation de l'erreur en langue étrangère. Coll. Didactique des langues, Nathan. 
Mazur-Palandre, A. (2015). Overcoming Preferred Argument Structure in written French: development, modality, text type. Written Language and Literacy, 18, 25-55.

Mazur-Palandre, A. et Jisa, H. (2013). La complexité lexicale des syntagmes nominaux : Une étude développementale. Enfance, 4, 359-371.

Mazur-Palandre, A., Abadie, R. et Bedoin, N. (2016). Étudiants dyslexiques à l'Université: Spécificités des difficulté ressenties et évaluation des déficits. In M. Habib (Ed.), 141-179. De Boeck.

Morken, F. et Helland, T. (2013). Writing in Dyslexia: Product and Process. Dyslexia, 19:131-148.

Mosenthal, P. (1985). Defining the expository discourse continuum, toward a taxinomy of expository text, Poetics, 14, 387-414.

Olive, T., Piolat, A. (2005). Le rôle de la mémoire de travail dans la production de textes, Psychologie Française, 50, 373-390.

Ortéga, É., et Lété, B. (2010). eManulex: Electronic version of Manulex and Manulex-infra databases. Retrieved from http://www.manulex.org.

Piolat. A. (2004). Approche cognitive de l'activité rédactionnelle et de son acquisition. Le rôle de la mémoire de travail, LINX (Linguistique Institut Nanterre Paris X), 51, 55-74.

Ravid, D. (2005). Emergence of linguistic complexity in later language development:evidence from expository text construction, In Ravid, D., Shyldkrot, H.B. (Eds.), Perspectives on language and language development. Essays in Honor of Ruth A. Berman, 337-355. Dordrecht: Kluwer Academic Publishers.

Ringard. J.-C. (2000). A propos de l'enfant dysphasique et de l'enfant dyslexique. Besoin spécifiques.

Swanson, H. L. , Hsieh. C.-J. (2009). Reading disabilities in adults: A selective meta-analyses of the literature. Review of Educational Research, 79(4), 1362-1390.

Valdois, S., Guinet, E., et Embs, J.-L. (Producer). EVADYS. Isbergues : Ortho Editions.

Zimmermann, P., et Fimm, B. (2012). Testbatterie zur Aufmerksamkeitsprüfung - Version Mobilität (Test battery for the assessment of attentional skills-Mobility version). Herzogenrath: Psytest.

Zorman, M., Lequette, C., Pouget, G. (2004). Un dépistage des difficultés de langage oral et des risques de dyslexie. Dans DM.-N. Metz-Lutz et al. (Eds.), Développement cognitif et troubles des apprentissages : évaluer, comprendre, rééduquer et prendre en charge, 245-270. Marseille: Solal.

\footnotetext{
${ }^{i}$ Corresponding author : audrey.mazur_palandre@ens-lyon.fr

ii Données 2012/2013 de la Mission Handicap de l'Université de Lyon.

iii ETUDYS (Accueil, intégration et accompagnement des ETUdiants DYSlexiques à l'université) et DYS'R'ABLE (Etudiants DYSlexiques à l'université : difficultés en Rédaction et en Anglais, évaluations et aides pour un accès équitaBLE aux conditions de réussite) : PEPS CNRS, LabEx ASLAN, laboratoires DDL et ICAR ; FLEXiDys (FLEXibilité lexicale, syntaxique et discursive des étudiants DYSlexiques) : ENS de Lyon.

${ }^{\text {iv }}$ Seul un étudiant déclare ne pas avoir été diagnostiqué mais le bilan confirme une dyslexie.

v Avec l'aide de l'équipe PANEL, ISH de Lyon.

${ }^{v i}$ Raphaëlle Abadie, Psychologue-Neuropsychologue au Centre d'Action Médico-Sociale Précoce de Décines (69).

vii Nathalie Bedoin, MCF, Université Lyon 2 et Laboratoire DDL (UMR 5596).

viii Vidéo créée pour le projet Spencer (fondation Spencer, USA), porteur Ruth Berman.

${ }^{\text {ix }}$ Filtre réalisé par Florence Chenu (Laboratoire DDL, UMR5596, CNRS et Université Lyon 2).

${ }^{x}$ Filtre réalisé par Florence Chenu (Laboratoire DDL, UMR5596, CNRS et Université Lyon 2).

${ }^{\mathrm{xi}} \mathrm{S}=$ significatif $/ \mathrm{NS}=$ non significatif $/ \mathrm{N}=$ Nombre d'individus $(21$ dyslexiques et 22 contrôles appariés)

${ }^{\text {xii }}$ Les exemples sont des extraits de textes d'étudiants dyslexiques; $\mathrm{TN}=$ texte narratif $/ \mathrm{TE}=$ texte expositif.
} 